\title{
MicroRNAs from the Planarian Schmidtea mediterranea: A model system for stem cell biology
}

\author{
DASARADHI PALAKODETI, ${ }^{1}$ MAGDA SMIELEWSKA, ${ }^{1}$ and BRENTON R. GRAVELEY \\ Department of Genetics and Developmental Biology, University of Connecticut Health Center, Farmington, Connecticut 06030-3301, USA
}

\begin{abstract}
MicroRNAs (miRNAs) are $\sim 22-n t$ RNA molecules that typically bind to the $3^{\prime}$ untranslated regions of target mRNAs and function to either induce mRNA degradation or repress translation. miRNAs have been shown to play important roles in the function of stem cells and cell lineage decisions in a variety of organisms, including humans. Planarians are bilaterally symmetric metazoans that have the unique ability to completely regenerate lost tissues or organs. This regenerative capacity is facilitated by a population of stem cells known as neoblasts. Planarians are therefore an excellent model system for studying many aspects of stem cell biology. Here we report the cloning and initial characterization of 71 miRNAs from the planarian Schmidtea mediterranea. While several of the $S$. mediterranea miRNAs are members of miRNA families identified in other species, we also identified a number of planarian-specific miRNAs. This work lays the foundation for functional studies aimed at addressing the role of these miRNAs in regeneration, cell lineage decisions, and basic stem cell biology.
\end{abstract}

Keywords: microRNA; Schmidtea mediterranea; planarians; stem cells

\section{INTRODUCTION}

Planarians are best known for their amazing regenerative capacity-even a fragment as small as $1 / 279^{\text {th }}$ the size of the original individual has the capacity to regenerate into an entire animal (Newmark and Sánchez Alvarado 2002). The key to the regenerative prowess of these creatures are neoblasts, a population of totipotent cells distributed throughout the organism (Newmark and Sánchez Alvarado 2002). Upon injury, the neoblasts migrate to the wound site, divide, and their progeny eventually differentiate to replace the missing structures. Neoblasts also function in homeostasis by serving to replace cells lost during normal cellular turnover. Thus, neoblasts are the planarian equivalent of stem cells, making these remarkable creatures an excellent model system for studying stem cell biology (Sánchez Alvarado 2006).

Planarians are also extraordinarily interesting from an evolutionary perspective because their precise relationship

\footnotetext{
${ }^{1}$ These authors contributed equally to this work.

Reprint requests to: Brenton R. Graveley, Department of Genetics and Developmental Biology, University of Connecticut Health Center, 263 Farmington Avenue, Farmington, CT 06030-3301, USA; e-mail: graveley@ neuron.uchc.edu; fax: (860) 679-8345.

Article published online ahead of print. Article and publication date are at http://www.rnajournal.org/cgi/doi/10.1261/rna.117206.
}

to other metazoans has not clearly been established. Planarians are bilaterally symmetric metazoans and are members of the phylum platyhelminthes (Newmark and Sánchez Alvarado 2002). Early studies classified planarians as acoelomates and, thus, placed them more distantly related to vertebrates than insects or nematodes (Adoutte et al. 1999, 2000). In contrast, molecular analyses based on ribosomal RNA sequences have placed planarians in the lophotrochozoan clade of protostomes (Adoutte et al. 1999, 2000). This classification suggests that planarians are actually more closely related to vertebrates than insects or nematodes. In fact, of the planarian ESTs that are homologous with other GenBank entries, 64\% encode proteins that are more similar to vertebrate genes than to invertebrate genes (Sánchez Alvarado et al. 2002). However, a more recent analysis of metazoan phylogeny that compared a common set of 50 genes from each organism analyzed concluded that platyhelminthes are more similar to nematodes than vertebrates or insects (Rokas et al. 2005). Soon, the genome sequence of the planarian Schmidtea mediterranea will be completed and this should provide a clearer picture of the precise relationship of planarians to other metazoans.

MicroRNAs (miRNAs) are $\sim 18-25$-nt long noncoding RNA molecules that play an important role in regulating gene expression by binding to mRNAs and silencing the 
expression of the encoded proteins (Valencia-Sanchez et al. 2006). miRNAs are synthesized as large primary miRNA (pri-miRNA) transcripts that contain the mature miRNAs embedded within distinct stem-loop structures. These stem-loops are sequentially processed by the RNaseIII enzymes Drosha and Dicer (Kim 2005) and the mature miRNAs are assembled into a miRNA Induced Silencing Complex (miRISC) containing a member of the Argonaute family of proteins. miRISC then associates with and silences target mRNAs (Valencia-Sanchez et al. 2006).

Though the first miRNAs were identified genetically (Lee et al. 1993; Reinhart et al. 2000), hundreds of plant and animal miRNAs have now been identified through molecular cloning and/or bioinformatic approaches (LagosQuintana et al. 2001; Lau et al. 2001; Lee and Ambros 2001; Aravin et al. 2003; Lagos-Quintana et al. 2003; Lai et al. 2003; Lim et al. 2003; Jones-Rhoades and Bartel 2004; Bentwich et al. 2005; Cummins et al. 2006). In animals, miRNAs function by imperfectly base-pairing with the $3^{\prime}$ untranslated regions of target mRNAs where they either induce mRNA degradation (Yekta et al. 2004; Bagga et al. 2005; Jing et al. 2005; Lim et al. 2005) or inhibit translation of the mRNA (Humphreys et al. 2005; Pillai et al. 2005; Petersen et al. 2006). It is thought that there are thousands of mRNAs that are subject to miRNA regulation in animals (Brennecke et al. 2005; Krek et al. 2005; Lewis et al. 2005; Xie et al. 2005), though relatively few miRNA:mRNA pairs have been experimentally validated to date. In cases where miRNA-mediated gene regulation has been validated, it is clear that miRNAs play criticial roles in many aspects of development and cell lineage decisions (Ambros 2004; He and Hannon 2004). To cite just a few examples, miRNAs have been shown to regulate developmental timing (Lee et al. 1993; Reinhart et al. 2000), cell proliferation (Brennecke et al. 2003), left-right asymmetry in neural development (Johnston and Hobert 2003), HOX gene expression (Naguibneva et al. 2006), dendritic spine development (Schratt et al. 2006), and early embryogenesis (Giraldez et al. 2006). miRNAs also play important roles in stem cell biology. For example, a number of miRNAs that are specifically expressed in human and mouse embryonic stem cells have been described (Houbaviy et al. 2003; Suh et al. 2004). Moreover, miRNAs have been shown to control the differentiation of hematopoietic stem cells in mice (Chen et al. 2004) and germline stem cells in Drosophila (Forstemann et al. 2005; Hatfield et al. 2005).

Given the precision by which cellular proliferation and differentiation must be coordinately controlled during regeneration, and the fact that in other organisms miRNAs have been implicated in the control of stem cell function and cell lineage decisions, it is likely that miRNAs will play a key role in regulating regeneration in planarians. Consistent with this hypothesis, two PIWI domain-containing proteins related to the Argonaute family have been iden- tified in S. mediterranea, and one of these, SMEDWI-2, is required for regeneration and homeostasis (Reddien et al. 2005b). Though this observation does not directly implicate the miRNA pathway, it does suggest that small RNAs play an important role in the control of homeostasis and regeneration in planarians. Thus, to fully understand how homeostasis and regeneration are regulated in planarians, it is important to identify planarian miRNAs and the target mRNAs they regulate.

As a first step toward investigating the role of miRNAs in regeneration, we report the cloning and characterization of 71 miRNAs from the planarian S. mediterranea Importantly, this is the first lophotrochozoan organism from which miRNAs have been reported. Though many of the $S$. mediterranea miRNAs belong to previously characterized miRNA families in other metazoans, several are unique to planarians. Interestingly, the majority of the conserved miRNAs are more similar to insect miRNAs than to miRNAs from either nematodes or vertebrates. This work now makes it possible to begin studying the roles of miRNAs in controlling regeneration, cell lineage decisions, and stem cell function in planarians.

\section{RESULTS AND DISCUSSION}

To begin to study the role of microRNAs in controlling regeneration, stem cell function, and cell lineage decisions in planarians, we set out to identify microRNAs from S. mediterranea. Our initial approach was to search the raw sequence reads of the unassembled $S$. mediterranea genome for sequences that are at least $90 \%$ identical to known human, Drosophila or Caenorhabditis elegans miRNAs. After identifying highly similar sequences, they were analyzed with Mfold 3.2 (Zuker 2003) to determine whether the sequence surrounding the putative miRNAs could fold into a stem-loop structure characteristic of pre-miRNAs. This approach yielded only 10 potential miRNAs. We therefore resorted to established cloning procedures (Lau et al. 2001). Briefly, 18-26-nt RNAs were isolated from both sexual and asexual strains of $S$. mediterranea, and specialized linkers were ligated to the $5^{\prime}$ and $3^{\prime}$ ends. These RNAs were reverse-transcribed, amplified by PCR, concatenated, cloned, and the sequence of over 2200 individual inserts was obtained.

To determine whether these sequences represent miRNAs, we first removed all sequences that were $<18 \mathrm{nt}$ (328 sequences) or $>25 \mathrm{nt}$ (five sequences) and subsequently analyzed the remaining sequences to identify and remove contaminating rRNA, tRNA, or sequences that perfectly matched spliced ESTs (421 sequences). The remaining sequences were then used to search the raw sequence reads of the unassembled $S$. mediterranea genome, and those that did not perfectly match the genome (685 sequences) were discarded. Due to the fact that miRNAs display heterogeneity at their ends, the remaining 820 sequences were 
assembled into groups of related sequences that differed slightly only at their $5^{\prime}$ or $3^{\prime}$ ends. Though heterogeneity was observed, only six instances of $5^{\prime}$ end heterogeneity was observed-the remaining 30 cases involved only $3^{\prime}$ end heterogeneity as expected, if these are authentic miRNAs. For each sequence we extracted 100 nt of genomic sequence flanking either side of the potential miRNA and tested whether stem-loop structures characteristic of known premiRNAs could be formed by using Mfold 3.2 (Zuker 2003). This led to a nonredundant set of 81 potential miRNAs represented by 773 individual sequences or $34 \%$ of all sequences we identified (Supplemental Fig. S1; Supplementary material can be found at http://genetics.uchc.edu/ graveley/supplemental/palakodetisupp.pdf). Importantly, of the 10 potential miRNAs identified by BLAST searching with the human, Drosophila or C. elegans miRNAs, all but two, $m i R-8$ and $m i R-13$, were present in these clones. Given the high degree of validation of the predicted miRNAs, we included $m i R-8$ and $m i R-13$ in our collection for a total of 83 potential miRNAs (Tables 1,2 ).

Previous studies have shown that, in addition to the mature miRNA, miRNA* strands, the complement of the miRNA derived from Dicer cleavage of the pre-mRNA can occasionally be cloned ( $\mathrm{Lim}$ et al. 2003). To determine whether any of the sequences we obtained corresponded to miRNA* strands, we compared the sequence of each premiRNA stem-loop with all others. This analysis revealed that 12 of the putative miRNA sequences actually corresponded to miRNA* strands (Table 1). Thus, we have identified a total of 71 potential miRNA genes from S. mediterranea (Supplemental Fig. S2; Supplemental materials are available online at http://genetics.uchc.edu/ graveley/supplemental/palakodetisupp.pdf).

We next determined whether any of the potential miRNAs we identified were similar to miRNAs in other organisms by searching miRBase (Griffiths-Jones et al. 2006) with the mature sequence of each putative miRNA. We considered miRNA pairs to be members of a family if two criteria were met. First, the seed regions, typically nucleotides 2-7 of the miRNA that serve to anchor the miRNA to the mRNA target (Lewis et al. 2005), had to be identical. Second, the mature miRNAs had to be located on the same side of the pre-miRNA stem-loops. Using these criteria, we found that 54 of the cloned miRNAs are members of other metazoan miRNA families (Fig. 1), while 17 appear to be unique to $S$. mediterranea. The conserved miRNA families include the lin-4 (Lee et al. 1993), let-7 (Reinhart et al. 2000), and bantam (Brennecke et al. 2003). Interestingly, only 14 of the miRNA families are conserved in vertebrates, insects, and roundworms (Fig. 2). Surprisingly, $\sim 80 \%$ of the conserved miRNAs have family members in insects, while only $\sim 50 \%$ have family members in nematodes and vertebrates, despite the fact that more miRNAs have been isolated from vertebrates and nematodes than from insects (Fig. 2). This finding is interesting in the context of the uncertainty regarding the phylogenetic relationship of planarians to other metazoans.

To further validate these candidate miRNAs, the expression of 39 miRNAs was examined in both sexual and asexual strains of $S$. mediterranea, as well as in a second planarian Dugesia tigrina, by Northern blot experiments. Twenty representative northerns are shown in Figure 3. The expression of 33 of the miRNAs tested was detected in $S$. mediterranea and all of these except for $l i n-4 c$ were also detected in D. tigrina. For $m i R-71 c$ and $m i R-278$, multiple bands were detected on the Northern blots. This is consistent with the cloning of multiple forms of these miRNAs (Table 1). Six of the miRNAs were undetectable by Northern blotting. Three of these, $m i R-10^{\star}, m i R-190 b^{\star}$, and $m i R-277 b^{*}$, are miRNA* strands and are therefore not expected to be detected in this manner. One planarianspecific miRNA, miR-749, was cloned four times and may be expressed at levels undetectable by Northern blotting. Two other miRNAs that were negative by northerns, $m i R-A$ and $m i R-B$, are also planarian-specific miRNAs but were cloned only once. These two miRNAs, along with five other planarian-specific miRNAs that were cloned only once and not tested by northerns are considered as potential miRNAs and will require additional validation before being promoted to being official miRNAs (Table 2). While it is possible that these sequences do not represent actual miRNAs, it is also possible that they are authentic miRNAs that are simply expressed at low levels. Regardless, these results demonstrate that the majority of the miRNAs we identified are not only expressed, but are also conserved in other planarians. Although we did observe differences in the number of miRNAs cloned from the sexual and asexual strains (Table 1), we did not observe any significant difference in expression between the two strains by Northern blot (Fig. 3). This discrepancy could be reflective of the overall quality of the miRNA libraries and/or cross hybridization of the probes to highly similar miRNAs. Nonetheless, these experiments clearly demonstrate that most, if not all, of these small RNAs represent authentic miRNAs.

Although we identified four members of the let-7 family, we did not clone any miRNAs that perfectly matched the sequence of the canonical let-7 (let-7a), which is $100 \%$ identical in all metazoans examined so far (Pasquinelli et al. 2000). Moreover, even BLAST searches of the S. mediterranea genome, which has been sequenced to $8 \times$ coverage, did not identify any sequence that perfectly matched that of let-7. Thus, it appears that $S$. mediterranea may not contain the canonical let-7 gene.

As is the case in other organisms, the miRNAs we identified frequently occur in clusters. Our ability to thoroughly assess the distribution of miRNAs throughout the genome was limited by the fact that the $S$. mediterranea genome has yet to be assembled. Nonetheless, we examined whether any of the contigs containing the miRNAs overlapped with one another. This allowed us to identify seven 
TABLE 1. MicroRNAs from Schmidtea mediterranea

\begin{tabular}{|c|c|c|c|c|c|c|c|}
\hline miRNA gene & Sequence & Length $^{\mathrm{a}}$ & Sexual $^{\mathrm{b}}$ & Asexual $^{\mathrm{b}}$ & Fold-back arm & Conservation $^{c}$ & Northern ${ }^{\mathrm{d}}$ \\
\hline bantam-a & UGAGAUCACUAUGAAAGCUGG & $21-22$ & 17 & 33 & $3^{\prime}$ & I & $\mathrm{Y}$ \\
\hline bantam-b & UGAGAUCACUGCGAAAGCUGAU & $21-22$ & 0 & 9 & $3^{\prime}$ & I & $\mathrm{Y}^{*}$ \\
\hline bantam-c & UGAGAUCAUUAUGAAAGCUUUU & 22 & 0 & 1 & $3^{\prime}$ & I & $Y^{*}$ \\
\hline let-7a & UGAGGUAGAAUGUUGGAUGACU & $21-22$ & 2 & 11 & $5^{\prime}$ & $\mathrm{V}, \mathrm{I}, \mathrm{N}$ & Y \\
\hline let-7b & UGAGGUAGAUUGUUGGAUGACU & $21-22$ & 2 & 6 & $5^{\prime}$ & $\mathrm{V}, \mathrm{I}, \mathrm{N}$ & $\mathrm{Y}^{*}$ \\
\hline $1 e t-7 b^{*}$ & CCAUUCAACUAUCUGUCUUCUC & 22 & 1 & 0 & $3^{\prime}$ & $\mathrm{N} / \mathrm{A}$ & N/D \\
\hline let-7c & UGAGGUAGUGACUCAAAAGGUU & 22 & 1 & 12 & $5^{\prime}$ & $\mathrm{V}, \mathrm{I}, \mathrm{N}$ & $\mathrm{N} / \mathrm{D}$ \\
\hline $\operatorname{lin}-4 a$ & UCCCUGAGACCUUCGACUGUGU & $20-22$ & 7 & 61 & $5^{\prime}$ & $\mathrm{V}, \mathrm{I}, \mathrm{N}$ & $\mathrm{Y}$ \\
\hline $\operatorname{lin}-4 b$ & UCCCUGAGACCAUUGACUGCAU & $20-22$ & 7 & 30 & $5^{\prime}$ & $\mathrm{V}, \mathrm{I}, \mathrm{N}$ & $\mathrm{Y}$ \\
\hline $\operatorname{lin}-4 b^{*}$ & GUAGUUUUUGGUAUCAGGAU & 20 & 0 & 3 & $3^{\prime}$ & $\mathrm{N} / \mathrm{A}$ & $\mathrm{N} / \mathrm{D}$ \\
\hline lin-4c & UCCCUGAGAUCAUAAUAUGCCU & 22 & 3 & 1 & $5^{\prime}$ & $\mathrm{V}, \mathrm{I}, \mathrm{N}$ & $\mathrm{Y}$ \\
\hline$m i R-1 a$ & UGGAAUGUCGAGAAAUAUGCAU & 12,22 & 3 & 5 & $3^{\prime}$ & $\mathrm{V}, \mathrm{I}, \mathrm{N}$ & Y \\
\hline $\operatorname{miR}-1 b$ & UGGAAUGUCGUGAAUUAUGGUC & $20,22,24$ & 32 & 148 & $3^{\prime}$ & $\mathrm{V}, \mathrm{I}, \mathrm{N}$ & Y \\
\hline $\operatorname{miR}-1 c$ & UGGAAUGUUGUGAAUAGUGUC & 21 & 5 & 9 & $3^{\prime}$ & $\mathrm{V}, \mathrm{I}, \mathrm{N}$ & Y \\
\hline $\operatorname{miR}-2 \mathrm{a}-1$ & UAUCACAGCCCCGCUUGGAACGCU & $20-24$ & 4 & 5 & $3^{\prime}$ & $\mathrm{I}, \mathrm{N}$ & Y \\
\hline $\operatorname{miR}-2 a-2$ & UAUCACAGCCCCGCUUGGAACGCU & $20-24$ & 4 & 5 & $3^{\prime}$ & $\mathrm{I}, \mathrm{N}$ & Y \\
\hline $\operatorname{miR}-2 \mathrm{a}-2^{*}$ & GUUCUACGGUGUUGUGAUAU & 20 & 0 & 1 & $5^{\prime}$ & $\mathrm{N} / \mathrm{A}$ & $\mathrm{Y}$ \\
\hline $\operatorname{miR}-2 b$ & UCACAGCCAAUUUUGAUGAGAU & 19,22 & 0 & 4 & $3^{\prime}$ & $\mathrm{I}, \mathrm{N}$ & $\mathrm{Y}^{*}$ \\
\hline $\operatorname{miR}-2 b^{*}$ & UCAUCAUUGUUGGUUGUCAG & 20 & 0 & 1 & $5^{\prime}$ & $\mathrm{N} / \mathrm{A}$ & $\mathrm{N} / \mathrm{D}$ \\
\hline $\operatorname{miR}-2 c$ & UCACAGCCAAAACUGAUGAUCU & 20-22 & 2 & 6 & $3^{\prime}$ & $\mathrm{I}, \mathrm{N}$ & Y \\
\hline$m i R-2 d$ & UCACAGCCAAAUUUGAUGUCC & $18,21,22$ & 48 & 26 & $3^{\prime}$ & $\mathrm{I}, \mathrm{N}$ & $\mathrm{Y}$ \\
\hline$m i R-7 a$ & UGGAAGACUAUUGAUUUAGUUGA & 23 & 0 & 1 & $5^{\prime}$ & $\mathrm{V}, \mathrm{I}$ & $\mathrm{N} / \mathrm{D}$ \\
\hline$m i R-7 b$ & UGGAAGACUGUCGAUUUCGUUGU & 23 & 1 & 4 & $5^{\prime}$ & $\mathrm{V}, \mathrm{I}$ & N/D \\
\hline$m i R-7 c$ & UGGAAGACUGAUGAUUUGCUGA & 22 & 0 & 4 & $5^{\prime}$ & $\mathrm{V}, \mathrm{I}$ & N/D \\
\hline $\operatorname{miR}-8$ & UAAUACUGUCAGGUAACGAUGCC & 23 & & & $3^{\prime}$ & $\mathrm{V}, \mathrm{I}$ & $\mathrm{Y}$ \\
\hline $\operatorname{miR}-10$ & AACCCUGUAGAUCCGAGUUUGA & 22 & 4 & 7 & $5^{\prime}$ & $\mathrm{V}, \mathrm{I}$ & $\mathrm{Y}$ \\
\hline $\operatorname{miR}-10^{*}$ & UCGAAUCUUCAAGGUGAA & 19,22 & 0 & 1 & $3^{\prime}$ & $\mathrm{N} / \mathrm{A}$ & $\mathrm{N}$ \\
\hline $\operatorname{miR}-12$ & UGAGUAUUCUAUCAGGAGUCGA & 22 & 0 & 1 & $5^{\prime}$ & I & $\mathrm{N} / \mathrm{D}$ \\
\hline $\operatorname{miR}-13$ & UAUCACAGUCAUGCUAAAGAGC & 22 & & & $3^{\prime}$ & I & N/D \\
\hline $\operatorname{miR}-31 a$ & AGGCAAGAUGUUGGCAUAACUGA & $20-24$ & 0 & 5 & $5^{\prime}$ & $\mathrm{V}, \mathrm{I}, \mathrm{N}$ & $\mathrm{Y}$ \\
\hline $\operatorname{miR}-31 b$ & AGGCAAGAUGCUGGCAUAGCUGA & 23 & 0 & 1 & $5^{\prime}$ & $\mathrm{V}, \mathrm{I}, \mathrm{N}$ & $\mathrm{Y}^{*}$ \\
\hline $\operatorname{miR}-36$ & UCACCGGGUAGACAUUCAUUA & 21 & 1 & 0 & $3^{\prime}$ & $\mathrm{N}$ & N/D \\
\hline $\operatorname{miR}-36^{*}$ & UGAUGCAUGCUACUUGGUUU & 20 & 0 & 1 & $5^{\prime}$ & $\mathrm{N} / \mathrm{A}$ & $\mathrm{N} / \mathrm{D}$ \\
\hline $\operatorname{miR}-61$ & UGACUAGAAAGUUCACUUACUGU & 21,23 & 0 & 5 & $3^{\prime}$ & $\mathrm{I}, \mathrm{N}$ & $\mathrm{N} / \mathrm{D}$ \\
\hline $\operatorname{miR}-67$ & UCACAACCUCCAUGAACGAGGGU & 23 & 1 & 0 & $3^{\prime}$ & $\mathrm{I}, \mathrm{N}$ & $\mathrm{N} / \mathrm{D}$ \\
\hline $\operatorname{miR}-71 a-1$ & UGAAAGACACGGGUAGUGAGAU & 21,22 & 1 & 23 & $5^{\prime}$ & $\mathrm{N}$ & $\mathrm{Y}$ \\
\hline $\operatorname{miR}-71 a-2$ & UGAAAGACACGGGUAGUGAGAU & $21,22,24$ & 1 & 24 & $5^{\prime}$ & $\mathrm{N}$ & Y \\
\hline $\operatorname{miR}-71 b$ & UGAAAGACACAGGUAGUGGGAC & $20-23$ & 1 & 21 & $5^{\prime}$ & $\mathrm{N}$ & $\mathrm{Y}$ \\
\hline $\operatorname{miR}-71 b^{*}$ & UCCUUCUAAUGUGUUUUUCG & 20 & 0 & 1 & $3^{\prime}$ & $\mathrm{N} / \mathrm{A}$ & $\mathrm{N} / \mathrm{D}$ \\
\hline $\operatorname{miR}-71 \mathrm{c}$ & UGAAAGACAUGGGUAGUGAGAU & $20-22$ & 5 & 13 & $5^{\prime}$ & $\mathrm{N}$ & $\mathrm{Y}$ \\
\hline $\operatorname{miR}-79$ & GUAAAGCUAAAUUACCAAAGUGC & 23,25 & 1 & 7 & $3^{\prime}$ & $\mathrm{I}, \mathrm{N}$ & N/D \\
\hline $\operatorname{miR}-87 a$ & UGAGCAAAGUUUCAAGUGUA & 20 & 0 & 1 & $3^{\prime}$ & $\mathrm{I}, \mathrm{N}$ & N/D \\
\hline $\operatorname{miR}-87 b$ & GUGAGCAAAGCUUCAAAUGAG & 21 & 0 & 1 & $3^{\prime}$ & $\mathrm{I}, \mathrm{N}$ & N/D \\
\hline $\operatorname{miR}-92$ & GAUUGCACUAGUUAAUUAUC & 20 & 0 & 1 & $3^{\prime}$ & I & $\mathrm{Y}$ \\
\hline $\operatorname{miR}-124 a$ & UAAGGCACGCGGUGAAUGCUU & 21 & 0 & 1 & $3^{\prime}$ & $\mathrm{V}, \mathrm{I}, \mathrm{N}$ & $\mathrm{Y}^{*}$ \\
\hline $\operatorname{miR}-124 b$ & UAAGGCACGCGGUGAAUGCUGA & 20,22 & 0 & 3 & $3^{\prime}$ & $\mathrm{V}, \mathrm{I}, \mathrm{N}$ & $\mathrm{Y}^{*}$ \\
\hline $\operatorname{miR}-124 c$ & UAAGGCACGCGGUGAAUGCCA & $19-22$ & 3 & 19 & $3^{\prime}$ & $\mathrm{V}, \mathrm{I}, \mathrm{N}$ & $\mathrm{Y}$ \\
\hline $\operatorname{miR}-124 c^{*}$ & GCGCUCACCUCGUGACCUUUGU & 21,22 & 1 & 2 & $5^{\prime}$ & $\mathrm{N} / \mathrm{A}$ & N/D \\
\hline $\operatorname{miR}-133$ & UUGGUCCCCAUCAACCAGCA & 20 & 0 & 1 & $3^{\prime}$ & $\mathrm{V}, \mathrm{I}$ & N/D \\
\hline $\operatorname{miR}-184$ & GACGGAGGUUUGCUAAGGAA & 19,20 & 0 & 2 & $3^{\prime}$ & $\mathrm{V}, \mathrm{I}$ & N/D \\
\hline $\operatorname{miR}-190 a$ & AGAUAUGUUUGGUUAAUUGGUGA & 23 & 0 & 1 & $5^{\prime}$ & V & N/D \\
\hline $\operatorname{miR}-190 a^{*}$ & ACCACUGACCGAGCAUAUCCA & $19-22$ & 7 & 8 & $3^{\prime}$ & $\mathrm{N} / \mathrm{A}$ & $\mathrm{Y}$ \\
\hline $\operatorname{miR}-190 b$ & UGAUAUGUUUGGUUUAUUGGUGA & 23 & 0 & 1 & $5^{\prime}$ & $\mathrm{V}$ & N/D \\
\hline $\operatorname{miR}-190 b^{*}$ & ACCAUUAGCCUAAUGUAUCGUGUA & 20,24 & 2 & 0 & $3^{\prime}$ & $\mathrm{N} / \mathrm{A}$ & $\mathrm{N}$ \\
\hline $\operatorname{miR}-219$ & UGAUUGUCCAUACGCAGUUCUCA & $21-23$ & 1 & 4 & $5^{\prime}$ & $\mathrm{V}, \mathrm{I}$ & $\mathrm{N} / \mathrm{D}$ \\
\hline $\operatorname{miR}-277 a$ & UAAAUGCACUAUCGGAUAUGAC & 22 & 1 & 0 & $3^{\prime}$ & 1 & Y \\
\hline $\operatorname{miR}-277 b$ & AAAAUGCAUUAUCUGGCCAAGA & 22 & 0 & 1 & $3^{\prime}$ & I & $Y^{*}$ \\
\hline $\operatorname{miR}-277 b^{*}$ & UUGAUCAGAAAUGCAGCUUC & 20 & 0 & 1 & $5^{\prime}$ & $\mathrm{N} / \mathrm{A}$ & $N$ \\
\hline $\operatorname{miR}-277 \mathrm{C}$ & UAAAUGCAUUAUCUGGUAUGAU & 22,23 & 0 & 3 & $3^{\prime}$ & 1 & $\mathrm{Y}^{*}$ \\
\hline $\operatorname{miR}-277 d$ & UAAAUGCAUUUAUCUGGCCAAG & 22 & 1 & 0 & $3^{\prime}$ & 1 & $\mathrm{Y}$ \\
\hline
\end{tabular}


TABLE 1. Continued

\begin{tabular}{|c|c|c|c|c|c|c|c|}
\hline miRNA gene & Sequence & Length $^{a}$ & Sexual ${ }^{b}$ & Asexual $^{b}$ & Fold-back arm & Conservation $^{\mathrm{c}}$ & Northern \\
\hline $\operatorname{miR}-278$ & UCGGUGGGAGUAACAUUCGA & $19-21$ & 6 & 26 & $3^{\prime}$ & I & $\mathrm{Y}$ \\
\hline $\operatorname{miR}-281$ & UGUCAUGGAUAUGCUCUUCAU & 19,21 & 1 & 1 & $3^{\prime}$ & $\mathrm{I}, \mathrm{N}$ & $\mathrm{Y}$ \\
\hline $\operatorname{miR}-281^{*}$ & UGAAGAGCUAUUCAUGAGGU & 20 & 1 & 0 & $5^{\prime}$ & $\mathrm{N} / \mathrm{A}$ & $\mathrm{Y}$ \\
\hline $\operatorname{miR}-745$ & UGCUGCCUGGUUAAGAGCUGUGU & 23 & 2 & 4 & $3^{\prime}$ & V & Y \\
\hline $\operatorname{miR}-746$ & UAGCACCAGGGUAUAUCGGGAU & 22 & 0 & 2 & $3^{\prime}$ & V & $\mathrm{N} / \mathrm{D}$ \\
\hline $\operatorname{miR}-747$ & UAAUCUCAUCUGGUAAUUGAAGU & 23 & 0 & 1 & $5^{\prime}$ & V & $\mathrm{N} / \mathrm{D}$ \\
\hline $\operatorname{miR}-748$ & UGGACGGAAGUGUAAUGAG & 19 & 0 & 1 & $3^{\prime}$ & $\mathrm{N} / \mathrm{A}$ & Y \\
\hline $\operatorname{miR}-749$ & GCUGGGAUGAGCCUCGGUGGU & 21 & 0 & 4 & $5^{\prime}$ & $\mathrm{N} / \mathrm{A}$ & $\mathrm{N}$ \\
\hline $\operatorname{miR}-750$ & UCAGAUCUAACUCUUCCAGUUCU & 23 & 1 & 1 & $3^{\prime}$ & $\mathrm{N} / \mathrm{A}$ & Y \\
\hline $\operatorname{miR}-751$ & CAUGUUUGAAUGGCCAUGACA & $20-21$ & 1 & 2 & $3^{\prime}$ & $\mathrm{N} / \mathrm{A}$ & $\mathrm{N} / \mathrm{D}$ \\
\hline $\operatorname{miR}-752$ & AGUCAGCAUUGGUGGUUU & 18 & 0 & 1 & $3^{\prime}$ & $\mathrm{N} / \mathrm{A}$ & N/D \\
\hline $\operatorname{miR}-753$ & GAGCUUGGAUUGUGAUCUCA & 20 & 0 & 1 & $5^{\prime}$ & $\mathrm{N} / \mathrm{A}$ & $\mathrm{N} / \mathrm{D}$ \\
\hline $\operatorname{miR}-754$ & GUUGCUUGGGGUUAUUACUA & 20 & 0 & 1 & $5^{\prime}$ & $\mathrm{N} / \mathrm{A}$ & $\mathrm{N} / \mathrm{D}$ \\
\hline $\operatorname{miR}-755$ & UGGAGCUAUUGUAUUUCACC & 18,20 & 1 & 1 & $5^{\prime}$ & $\mathrm{N} / \mathrm{A}$ & Y \\
\hline $\operatorname{miR}-756$ & CGAUAUGUGGUAAUUUGGAUGA & 22 & 5 & 0 & $5^{\prime}$ & $\mathrm{N} / \mathrm{A}$ & $\mathrm{Y}$ \\
\hline
\end{tabular}

'Variations in length are due to 5' and 3' heterogeneity observed during miRNA cloning. The sequence of the most frequently cloned isoform for each miRNA is shown.

${ }^{\mathrm{b}}$ The number of times each miRNA was cloned from the sexual and asexual strains is indicated.

${ }^{c}$ Organisms in which homologous miRNAs were identified are indicated. V, vertebrate; I, insect; N, nematode; N/A, no homolog identified.

${ }^{\mathrm{d}}$ The results of Northern blots are shown. $\mathrm{Y}$, positive northern result; $\mathrm{Y}^{*}$, sequence of miRNA is similar enough to another miRNA that was detected by Northern blotting in which cross-hybridization is possible; $N$, negative northern result; N/D, Northern blot not performed.

clusters that together account for 19 of the miRNAs or miRNA $^{\star}$ strands we identified. The largest cluster contains three stem-loop structures encoding the cloned miRNAs $m i R-71 b, m i R-71 b^{*}, m i R-2 d, m i R-752$, as well as a fourth stem-loop containing one of the predicted miRNAs, miR-13 (Fig. 4). We identified three additional clusters that each contain, one $m i R-2$ family member and one $m i R-71$ family member. Each of these clusters adopts similar secondary structures, yet displays little sequence similarity to one another aside from the mature miRNAs (Fig. S3A-D). In fact, two of these clusters, miR-71a-1/miR-2a-1 and $m i R-71 a-2 / m i R-2 a-2$, contain identical miRNAs. We also identified two clusters that contain two members of the miR-277 family each (Fig. S3E,F): One cluster contains $m i R-277 a$ and $m i R-277 b$, while the other contains $m i R-277 c$ and $m i R-277 d$. For both the miR-71/miR-2 and miR-277 clusters, it is likely that these miRNAs arose by gene duplication events followed by sequence divergence. For all of these clusters, it is likely that the linked miRNAs are expressed as a single pri-miRNA.

The collection of $S$. mediterranea miRNAs reported here provides the foundation to begin exploring the miRNAmediated gene regulatory networks that are involved in controlling homeostasis and regeneration. Determining the expression patterns and predicting potential target mRNAs for each of these miRNAs should point toward potential miRNA:mRNA pairs that could play a role in regulating regeneration. Although thousands of $S$. mediterranea ESTs have been cloned (Sánchez Alvarado et al. 2002; Zayas et al. 2005), accurate and robust miRNA target prediction must await assembly of the genome so that a complete, high quality $3^{\prime}$ UTR data set can be compiled. Nonetheless, once

TABLE 2. Potential MicroRNAs from Schmidtea mediterranea

\begin{tabular}{|c|c|c|c|c|c|c|c|}
\hline miRNA gene & Sequence & Length & Sexual $^{\mathrm{a}}$ & Asexual $^{\mathrm{a}}$ & Fold-back arm & Conservation $^{\mathrm{b}}$ & Northern $^{\mathrm{c}}$ \\
\hline $\operatorname{miR}-A$ & CACUCCUGCCCAAUCCCUUUC & 21 & 0 & 1 & $5^{\prime}$ & N/A & $\mathrm{N}$ \\
\hline $\operatorname{miR}-B$ & GGUAGGCAUAGUCUGUUGGUUAC & 23 & 0 & 1 & $5^{\prime}$ & $\mathrm{N} / \mathrm{A}$ & $\mathrm{N}$ \\
\hline $\operatorname{miR}-C$ & GUCCCACCUGAGAUGCCA & 18 & 0 & 1 & $3^{\prime}$ & N/A & N/D \\
\hline $\operatorname{miR}-D$ & GGGGUGCAAGCUCUUGAUUGAAGUC & 25 & 0 & 1 & $3^{\prime}$ & N/A & N/D \\
\hline miR-Ea & UGACCACUGGUGCGAUCCUGC & 21 & 0 & 1 & $3^{\prime}$ & N/A & N/D \\
\hline$m i R-E b$ & UGACCAUGGACGCGACCCUAC & 21 & 0 & 1 & $3^{\prime}$ & $\mathrm{N} / \mathrm{A}$ & N/D \\
\hline $\operatorname{miR}-F$ & CCUUACGGCCCCUAAACCUUAUU & 23 & 0 & 1 & $3^{\prime}$ & N/A & N/D \\
\hline $\operatorname{miR}-G$ & GAUUGCACUCAAUUAUGGUCAGA & 23 & 1 & 0 & $3^{\prime}$ & $\mathrm{N} / \mathrm{A}$ & N/D \\
\hline
\end{tabular}

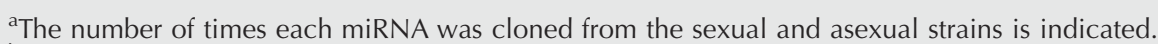

${ }^{b}$ These miRNAs are not similar to miRNAs from other organisms.

${ }^{\mathrm{C}}$ The results of Northern blots are shown. $\mathrm{N}$, negative northern result; $\mathrm{N} / \mathrm{D}$, Northern blot not performed. 
bantam family

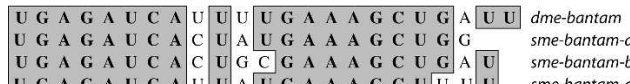

let-7 family

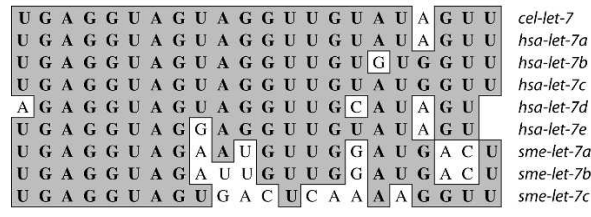

lin-4 family

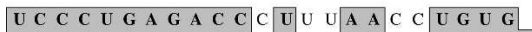
U C C C U A A

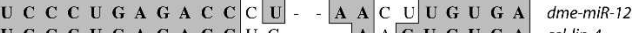

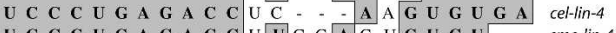

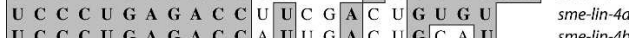

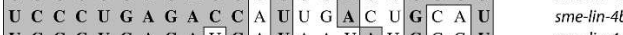

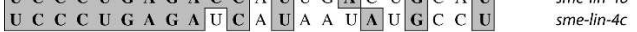

miR-1 family

U G G A A U G U A A A G A A G U A U G U G G A A U G G U G $G A U$ G U A A $A$ A $A$ G U U G U

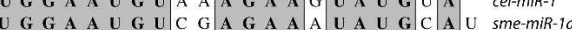
U G G A A U G U C G U G A A U U A U G G U C sme-miR-ib

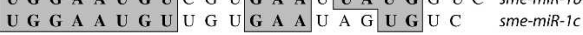

miR-2 family

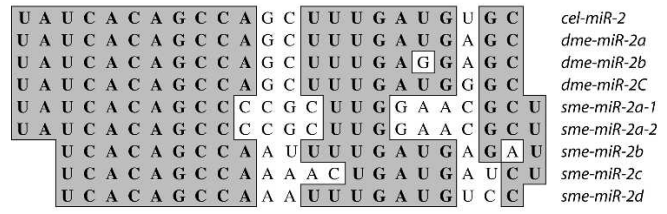

miR-7 family

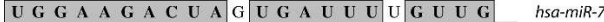
U G G A A G A C C U A $G$ G U G U G G A $A$ G A C U A U U G A U U A G U U G U G
$\mathbf{U}$

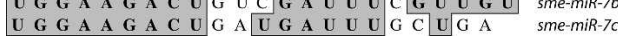

miR-8 family

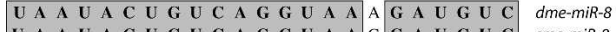

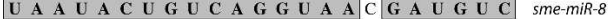

miR-10 family

\begin{tabular}{|c|c|}
\hline 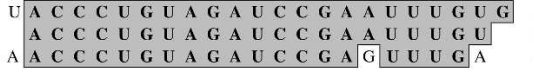 & $\begin{array}{l}\text { hsa-mik-10a } \\
\text { dme-miR-10 } \\
\text { sme-miR-10 }\end{array}$ \\
\hline \multicolumn{2}{|l|}{ miR-12 family } \\
\hline 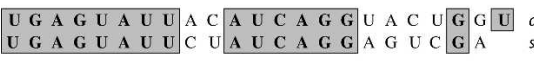 & $\begin{array}{l}\text { dme-miR-12 } \\
\text { sme-miR-12 }\end{array}$ \\
\hline \multicolumn{2}{|l|}{ miR-13 family } \\
\hline 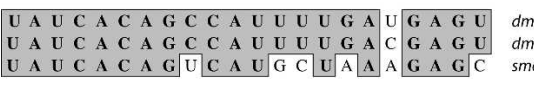 & $\begin{array}{l}\text { me-miR-13a } \\
\text { me-miR-136 } \\
\text { ne-miR-13 }\end{array}$ \\
\hline \multicolumn{2}{|l|}{ miR-22 family } \\
\hline 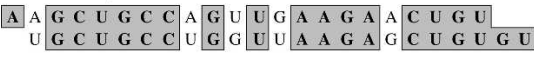 & $\begin{array}{l}\text { hsa-miR-22 } \\
\text { sme-miR-761 }\end{array}$ \\
\hline \multicolumn{2}{|l|}{ miR-29 family } \\
\hline 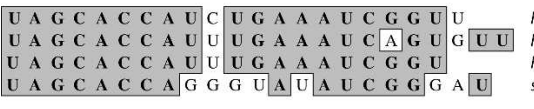 & $\begin{array}{l}\text { hsa-miR-29a } \\
\text { hsa-miR-29b } \\
\text { hsa-miR-29c } \\
\text { sme-miR-762 }\end{array}$ \\
\hline \multicolumn{2}{|l|}{ miR-31 family } \\
\hline 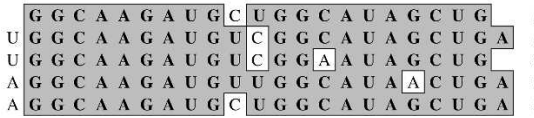 & $\begin{array}{l}\text { hsa-miR-31 } \\
\text { dme-miR-31a } \\
\text { dme-miR-31b } \\
\text { sme-miR-31a } \\
\text { sme-miR-31b }\end{array}$ \\
\hline
\end{tabular}

miR-36 family

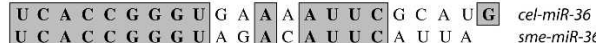

miR-61 family

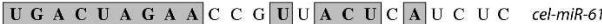
U G A C U A G A A A G U U C A C U U A C U G U sme-miR-6!

miR-71 family

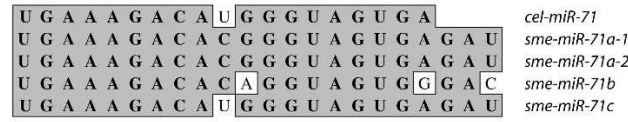

miR-79 family

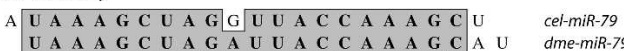

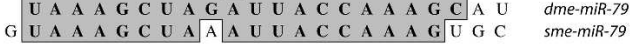

miR-87 family

G U G A G G C A A A A G G U U U U C C A

U U G A G C C A A A A A U U U C A G

U G A G C A A A G U U U C A A

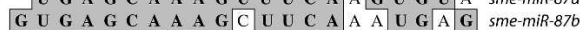

miR-92 family

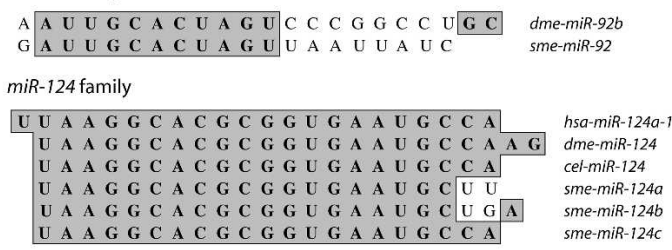

miR-133 family

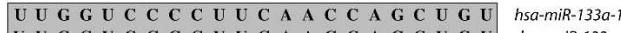

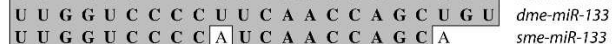

miR-184 family

U G G A C G G A G A A C U G A U A A G G G U ha-miR-184

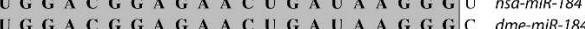

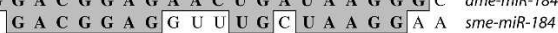

miR-190 family

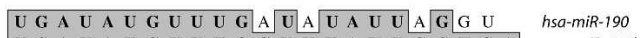
U G A U A U G U U U G G U U U A U U G G U G $\mathbf{A}$ sme-miR-1906 $\Lambda$ G A U A U G U U U G G U U $\triangle$ A A U G G U G A sme-mir-190a

miR-216 family

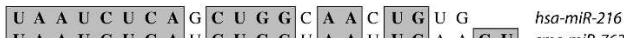

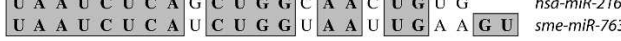

miR-219 family

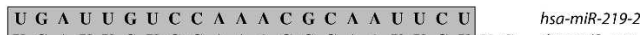
U G A U U G U C C C A A A C G C A A U U C U U G

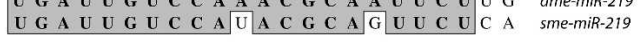

miR-277 family

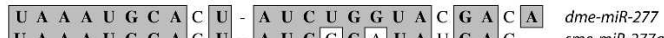

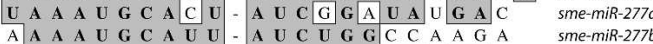

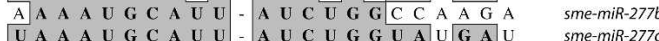

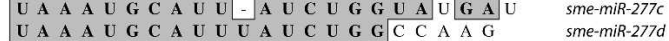

miR-278 family

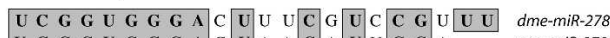
\begin{tabular}{lllllllllll}
\hline & C C G G U G G G G A \\
\hline
\end{tabular}

miR-281 family

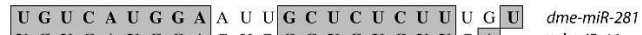

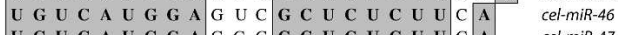

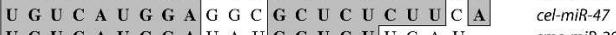

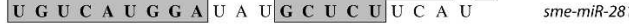

miR-307 family

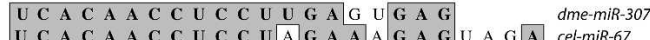

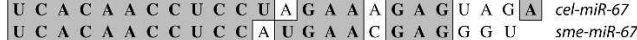

FIGURE 1. MicroRNA families. Alignments of $S$. mediterranea miRNAs with family members from human, Drosophila, or C. elegans miRNAs were performed with ClustalW. In some instances, only one representative from each family is shown. 


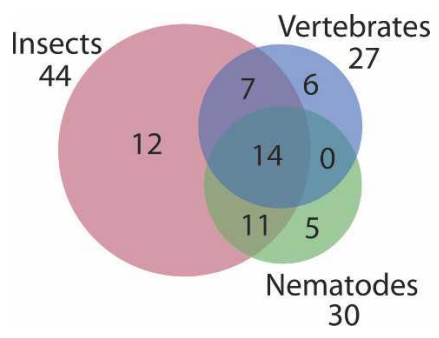

FIGURE 2. Relationships between metazoan miRNAs. A Venn diagram depicting the number of $S$. mediterranea miRNAs that have family members in vertebrates, insects, or nematodes.

potential targets are identified, they can be validated through a combination of RNA interference (Newmark et al. 2003; Reddien et al. 2005a), transgenic (GonzalezEstevez et al. 2003), antisense (Leaman et al. 2005), and microarray approaches. Such studies should provide insight into how miRNA-mediated gene regulation contributes to the control of homeostasis and regeneration in planarians. This will also increase our understanding of how stem cells are regulated in planarians, which may very well be similar in humans.

\section{MATERIALS AND METHODS}

\section{Planarian culturing}

Asexual and sexual strains of $S$. mediterranea were kind gifts from A. Sánchez Alvarado and P. Newmark. S. mediterranea was maintained at room temperature $\left(21-22^{\circ} \mathrm{C}\right)$ in $\mathrm{dd}_{2} \mathrm{O}$ supplemented with $1.6 \mathrm{mM} \mathrm{NaCl}, 1.0 \mathrm{mM} \mathrm{CaCl}_{2}, 1.0 \mathrm{mM} \mathrm{MgSO}_{4}, 0.1$

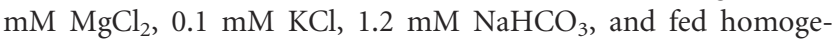
nized beef liver. Dugesia tigrina were obtained from Carolina Biological Supply and were maintained in Poland Spring water at room temperature. All animals were starved for one week prior to any experiments.

\section{Small RNA cloning}

Small RNA isolation and cloning from S. mediterranea were done essentially as described previously (Lau et al. 2001). Total RNA was separately isolated from sexual and asexual animals using Trizol as described by the manufacturer (Invitrogen). Trace amounts of ${ }^{32} \mathrm{P}-5^{\prime}$-end-labeled markers of 18 (AGCGUGUAGGG AUCCAAA) and 24 (GGCCAACGUUCUCAACAAUAGUGA) nt were added to each RNA sample and then separated on a $15 \%$ denaturing polyacrylamide gel. RNAs ranging from 18 to $24 \mathrm{nt}$ were excised from the gel and eluted overnight in $0.4 \mathrm{M} \mathrm{NaCl}$ at $4^{\circ} \mathrm{C}$. The eluted RNA was precipitated with two volumes of ethanol in the presence of $1 \mu \mathrm{g} / \mathrm{mL}$ of glycogen as carrier. The purified RNA was ligated to the modban linker (AMP-5' $\mathrm{p}-5^{\prime} \mathrm{p}-\mathrm{CT}$ GTAGGCACCATCAATdi-deoxyC-3') (Integrated DNA Technology) with $1 \mathrm{U} / \mu \mathrm{L}$ T4 RNA ligase (NEB) for $2 \mathrm{~h}$ at room temperature in a reaction mixture contatining $50 \mathrm{mM}$ HEPES $\mathrm{pH} 8.3$, $5 \mathrm{mM} \mathrm{MgCl} 2,3.3 \mathrm{mM}$ DTT, $10 \mu \mathrm{g} / \mathrm{mL} \mathrm{BSA}$, and $8.3 \%$ glycerol. The ligation mixture was separated on a $10 \%$ denaturing poly- acrylamide gel, and the ligated products excised and eluted as before. The RNAs were then ligated to $100 \mu \mathrm{M} 5^{\prime}$ linker oligo (5'-ATCGTrArGrGrCrArCrCrUrGrArArA-3') (Intergrated DNA Technology) in a reaction containing $0.4 \mathrm{mM} \mathrm{ATP}, 1 \mathrm{U} / \mu \mathrm{L}$ T4 RNA ligase for $6 \mathrm{~h}$ at room temperature. The ligation mixture was again resolved on a $10 \%$ denaturing polyacrylamide gel and the ligated products excised and eluted as before. The ligated RNA molecules were converted to cDNA with SuperScriptII (Invitrogen) and the Reverse Transcription (RT) primer (5'-ATTGATGG TGCCTACAG-3'). Subsequently, the products were amplified by PCR using the RT primer and the $5^{\prime}$ primer $\left(5^{\prime}\right.$-ATCGTAGGCA CCTGAAA- $3^{\prime}$ ) using the following protocol: $96^{\circ} \mathrm{C}, 1 \mathrm{~min}, 26$ cycles of $96^{\circ} \mathrm{C} 10 \mathrm{sec}, 50^{\circ} \mathrm{C} 1 \mathrm{~min}, 72^{\circ} \mathrm{C} 20 \mathrm{sec}$, followed by a 3 -min incubation at $72^{\circ} \mathrm{C}$. The PCR products were phenol-extracted, ethanol-precipitated, and digested with BanI $(20 \mathrm{U} / \mu \mathrm{L})$. The digested products were concatemerized by incubation with $\mathrm{T} 4$ DNA ligase (NEB) and the reaction mixture was resolved on a $2 \%$ low-melting agarose gel. Concatemers ranging from 300 to $600 \mathrm{bp}$ were eluted from the gel, tailed with Taq DNA polymerase, and cloned into pPCR-2.1-TOPO (Invitrogen). Colony PCR was performed to identify clones containing inserts $>2300-600$ bp in size and those clones were sequenced.

\section{Bioinformatic analysis}

The sequences of individual inserts between 18 and $25 \mathrm{nt}$ were used in local BLAST searches to screen databases of S. mediterranea
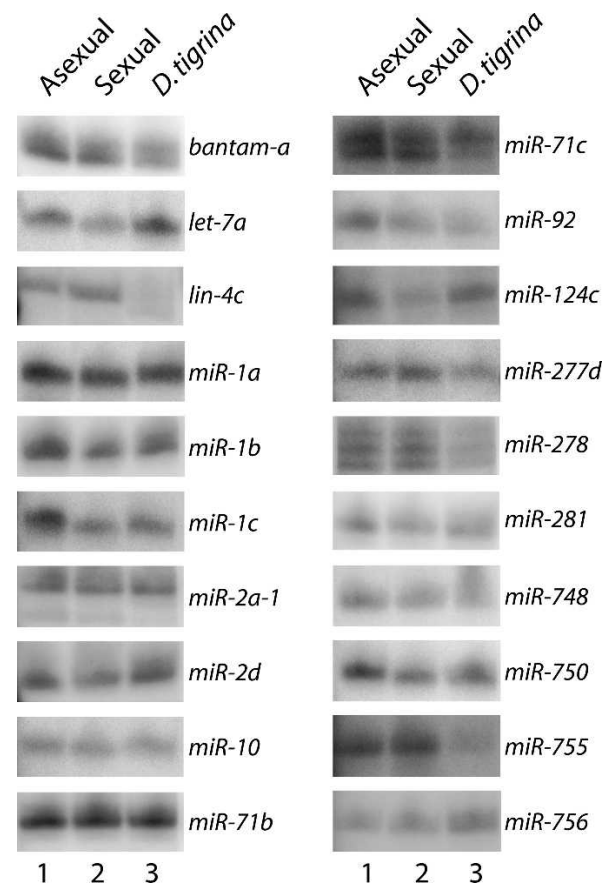

FIGURE 3. Northern blot analysis of miRNA expression. Fifty micrograms of total RNA isolated from both sexual and asexual strains of S. mediterranea and another planarian, Dugesia tigrina, were resolved on $15 \%$ denaturing polyacrylamide gels and transferred to membranes. Ethidium bromide staining of the gels indicated that equal amounts of RNA were loaded into each well. The blots were probed with ${ }^{32} \mathrm{P}, 5^{\prime}$-end-labeled oligonucleotides complementary to the miRNAs indicated. 


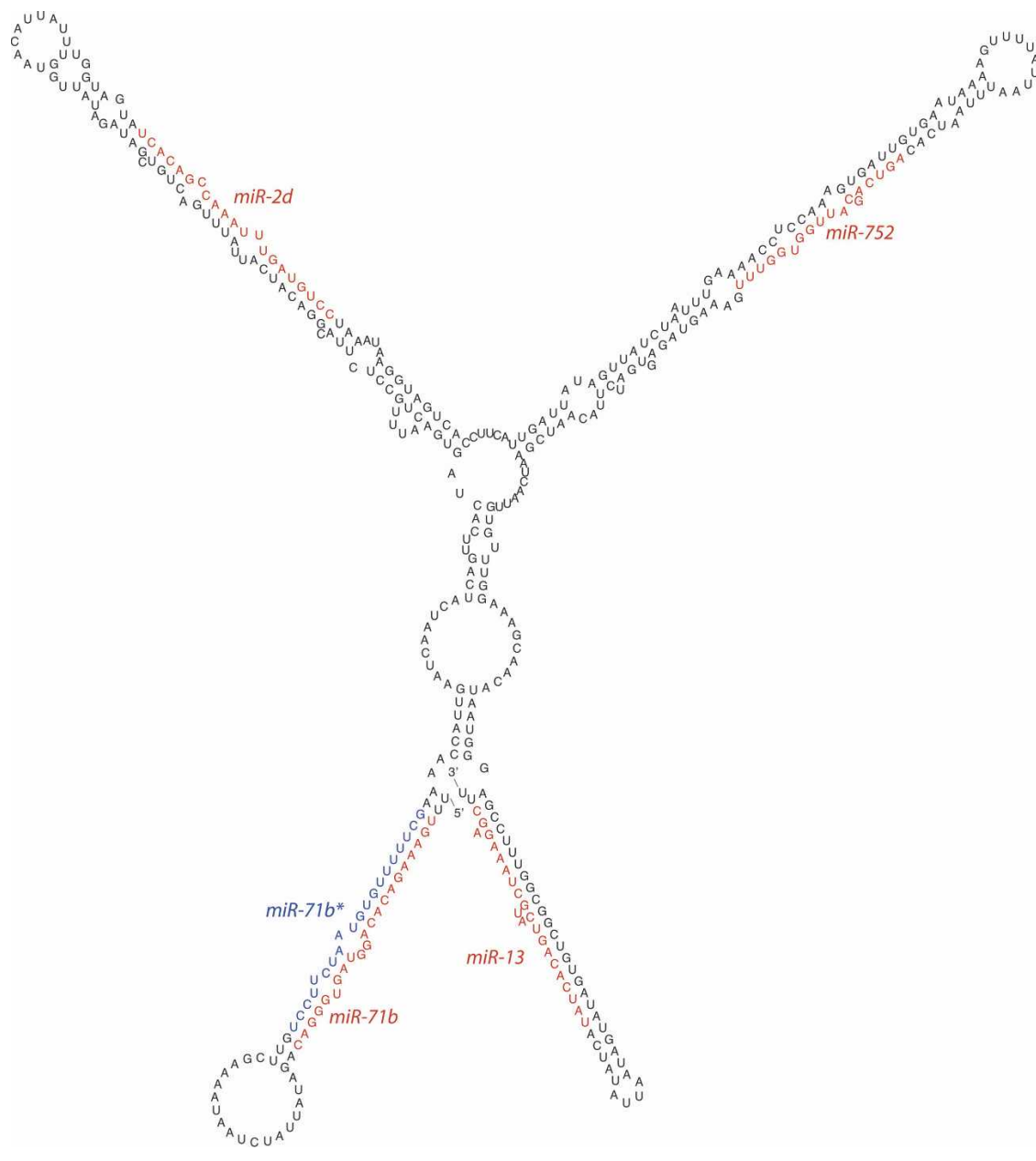

FIGURE 4. A S. mediterranea miRNA cluster. The predicted secondary structure of a premiRNA containing $m i R-71 b, m i R-71 b^{*}, m i R-2 d, m i R-752$, and a predicted miRNA, $m i R-13$. The sequence of the mature miRNAs is shown in red and the miRNA* strands that were identified are shown in blue. resulting contigs containing the miRNA clusters were reexamined with MFold.

\section{Northern blots}

Total RNA was extracted using TRIzol (Invitrogen) according to the manufacturer's protocol. Fifty micrograms of total RNA was loaded in each lane and resolved on $15 \%$ denaturing polyacrylamide gel. The gels were stained with ethidium bromide to confirm equal loading of the samples and then transferred onto a GeneScreen Plus membrane (Perkin Elmer), UV cross-linked, and baked for an hour at $80^{\circ} \mathrm{C}$. Oligonucleotide probes complementary to the miRNAs were ${ }^{32} \mathrm{P}$-end-labeled with $\mathrm{T} 4$ kinase and hybridized overnight at $42^{\circ} \mathrm{C}$ in $7 \%$ SDS and $0.2 \mathrm{M} \mathrm{Na}_{2} \mathrm{PO}_{4}, \mathrm{pH}$ 7.0. The blots were washed in $2 \times$ SSC, $0.1 \%$ SDS at room temperature, and visualized with a Cyclone Phosphoimager (Perkin Elmer).

\section{ACKNOWLEDGMENTS}

We thank Alejandro Sánchez Alvarado and Phil Newmark for providing the sexual and asexual S. mediterranea animals. We thank Amy Pasquinelli, Erik Sontheimer, Asis Das, Gene Yeo, and members of the Graveley lab for comments on the manuscript and discussions. This work was generously supported by the Raymond and Beverly Sackler Fund for the Arts and Sciences to B.R.G.

Received April 18, 2006; accepted May 26, 2006.
rRNA, ESTs, and the entire collection of unassembled genomic sequence reads produced by the Genome Sequencing Center at Washington University School of Medicine in St. Louis. Sequences that did not perfectly match the genome corresponded to rRNA, EST, or other repetitive sequence elements were discarded from further analysis. This filtering step did not remove sequences that reside within introns. The remaining sequences were compared with one another to identify groups of identical or highly related sequences. Roughly $100 \mathrm{nt}$ of genomic DNA flanking these sequences were extracted and analyzed with Mfold 3.2 (Zuker 2003) for the potential to adopt a stem-loop conformation typical of pre-miRNAs, and those that did not were discarded. The remaining sequences were then screened against miRBase (GriffithsJones et al. 2006) using SSEARCH to identify miRNA family members in other species. miRNA clusters were identified by first extracting all of the unassembled reads that perfectly corresponded to each miRNA. All of the reads for each miRNA were assembled into contigs using the CAP sequence assembly program (Huang 1996). Subsequently, all contigs were analyzed with the CAP sequence assembly program to identify further overlaps. The

\section{REFERENCES}

Adoutte, A., Balavoine, G., Lartillot, N., and de Rosa, R. 1999. Animal evolution. The end of the intermediate taxa? Trends Genet. 15: 104-108.

Adoutte, A., Balavoine, G., Lartillot, N., Lespinet, O., Prud'homme, B., and de Rosa, R. 2000. The new animal phylogeny: Reliability and implications. Proc. Natl. Acad. Sci. 97: 4453-4456.

Ambros, V. 2004. The functions of animal microRNAs. Nature 431: 350-355.

Aravin, A.A., Lagos-Quintana, M., Yalcin, A., Zavolan, M., Marks, D., Snyder, B., Gaasterland, T., Meyer, J., and Tuschl, T. 2003. The small RNA profile during Drosophila melanogaster development. Dev. Cell 5: 337-350.

Bagga, S., Bracht, J., Hunter, S., Massirer, K., Holtz, J., Eachus, R., and Pasquinelli, A.E. 2005. Regulation by let-7 and lin-4 miRNAs results in target mRNA degradation. Cell 122: 553-563.

Bentwich, I., Avniel, A., Karov, Y., Aharonov, R., Gilad, S., Barad, O., Barzilai, A., Einat, P., Einav, U., Meiri, E., et al. 2005. Identification of hundreds of conserved and nonconserved human microRNAs. Nat. Genet. 37: 766-770.

Brennecke, J., Stark, A., Russell, R.B., and Cohen, S.M. 2005. Principles of microRNA-target recognition. PLoS Biol. 3: e85. 
Brennecke, J., Hipfner, D.R., Stark, A., Russell, R.B., and Cohen, S.M. 2003. bantam encodes a developmentally regulated microRNA that controls cell proliferation and regulates the proapoptotic gene hid in Drosophila. Cell 113: 25-36.

Chen, C.Z., Li, L., Lodish, H.F., and Bartel, D.P. 2004. MicroRNAs modulate hematopoietic lineage differentiation. Science 303: 8386.

Cummins, J.M., He, Y., Leary, R.J., Pagliarini, R., Diaz, L.A.J., Sjoblom, T., Barad, O., Bentwich, Z., Szafranska, A.E., Labourier, E., et al. 2006. The colorectal microRNAome. Proc. Natl. Acad. Sci. 103: 3687-3692.

Forstemann, K., Tomari, Y., Du, T., Vagin, V.V., Denli, A.M., Bratu, D.P., Klattenhoff, C., Theurkauf, W.E., and Zamore, P.D. 2005. Normal microRNA maturation and germ-line stem cell maintenance requires Loquacious, a double-stranded RNA-binding domain protein. PLoS Biol. 3: e236.

Giraldez, A.J., Mishima, Y., Rihel, J., Grocock, R.J., Van Dongen, S., Inoue, K., Enright, A.J., and Schier, A.F. 2006. Zebrafish MiR-430 promotes deadenylation and clearance of maternal mRNAs. Science 312: 75-79.

Gonzalez-Estevez, C., Momose, T., Gehring, W.J., and Salo, E. 2003. Transgenic planarian lines obtained by electroporation using transposon-derived vectors and an eye-specific GFP marker. Proc. Natl. Acad. Sci. 100: 14046-14051.

Griffiths-Jones, S., Grocock, R.J., van Dongen, S., Bateman, A., and Enright, A.J. 2006. miRBase: MicroRNA sequences, targets and gene nomenclature. Nucleic Acids Res. 34: D140-D144.

Hatfield, S.D., Shcherbata, H.R., Fischer, K.A., Nakahara, K., Carthew, R.W., and Ruohola-Baker, H. 2005. Stem cell division is regulated by the microRNA pathway. Nature 435: 974978.

He, L. and Hannon, G.J. 2004. MicroRNAs: Small RNAs with a big role in gene regulation. Nat. Rev. Genet. 5: 522-531.

Houbaviy, H.B., Murray, M.F., and Sharp, P.A. 2003. Embryonic stem cell-specific MicroRNAs. Dev. Cell 5: 351-358.

Huang, X. 1996. An improved sequence assembly program. Genomics 33: $21-31$.

Humphreys, D.T., Westman, B.J., Martin, D.I., and Preiss, T. 2005. MicroRNAs control translation initiation by inhibiting eukaryotic initiation factor 4E/cap and poly(A) tail function. Proc. Natl. Acad. Sci. 102: 16961-16966.

Jing, Q., Huang, S., Guth, S., Zarubin, T., Motoyama, A., Chen, J., Di Padova, F., Lin, S.C., Gram, H., and Han, J. 2005. Involvement of microRNA in AU-rich element-mediated mRNA instability. Cell 120: 623-634.

Johnston, R.J. and Hobert, O. 2003. A microRNA controlling left/ right neuronal asymmetry in Caenorhabditis elegans. Nature 426: $845-849$.

Jones-Rhoades, M.W. and Bartel, D.P. 2004. Computational identification of plant microRNAs and their targets, including a stressinduced miRNA. Mol. Cell 14: 787-799.

Kim, V.N. 2005. MicroRNA biogenesis: Coordinated cropping and dicing. Nat. Rev. Mol. Cell Biol. 6: 376-385.

Krek, A., Grun, D., Poy, M.N., Wolf, R., Rosenberg, L., Epstein, E.J., MacMenamin, P., da Piedade, I., Gunsalus, K.C., Stoffel, M., et al. 2005. Combinatorial microRNA target predictions. Nat. Genet. 37: 495-500.

Lagos-Quintana, M., Rauhut, R., Lendeckel, W., and Tuschl, T. 2001. Identification of novel genes coding for small expressed RNAs. Science 294: 853-858.

Lagos-Quintana, M., Rauhut, R., Meyer, J., Borkhardt, A., and Tuschl, T. 2003. New microRNAs from mouse and human. RNA 9: $175-179$.

Lai, E.C., Tomancak, P., Williams, R.W., and Rubin, G.M. 2003. Computational identification of Drosophila microRNA genes. Genome Biol. 4: R42.

Lau, N.C., Lim, L.P., Weinstein, E.G., and Bartel, D.P. 2001. An abundant class of tiny RNAs with probable regulatory roles in Caenorhabditis elegans. Science 294: 858-862.
Leaman, D., Chen, P.Y., Fak, J., Yalcin, A., Pearce, M., Unnerstall, U., Marks, D.S., Sander, C., Tuschl, T., and Gaul, U. 2005. Antisensemediated depletion reveals essential and specific functions of microRNAs in Drosophila development. Cell 121: 1097-1108.

Lee, R.C. and Ambros, V. 2001. An extensive class of small RNAs in Caenorhabditis elegans. Science 294: 862-864.

Lee, R.C., Feinbaum, R.L., and Ambros, V. 1993. The C. elegans heterochronic gene lin-4 encodes small RNAs with antisense complementarity to lin-14. Cell 75: 843-854.

Lewis, B.P., Burge, C.B., and Bartel, D.P. 2005. Conserved seed pairing, often flanked by adenosines, indicates that thousands of human genes are microRNA targets. Cell 120: 15-20.

Lim, L.P., Lau, N.C., Weinstein, E.G., Abdelhakim, A., Yekta, S., Rhoades, M.W., Burge, C.B., and Bartel, D.P. 2003. The microRNAs of Caenorhabditis elegans. Genes \& Dev. 17: 991-1008.

Lim, L.P., Lau, N.C., Garrett-Engele, P., Grimson, A., Schelter, J.M., Castle, J., Bartel, D.P., Linsley, P.S., and Johnson, J.M. 2005. Microarray analysis shows that some microRNAs downregulate large numbers of target mRNAs. Nature 433: 769-773.

Naguibneva, I., Ameyar-Zazoua, M., Polesskaya, A., Ait-Si-Ali, S., Groisman, R., Souidi, M., Cuvellier, S., and Harel-Bellan, A. 2006. The microRNA miR-181 targets the homeobox protein Hox-A11 during mammalian myoblast differentiation. Nat. Cell Biol. 8: 278-284.

Newmark, P.A. and Sánchez Alvarado, A. 2002. Not your father's planarian: A classic model enters the era of functional genomics. Nat. Rev. Genet. 3: 210-219.

Newmark, P.A., Reddien, P.W., Cebria, F., and Sánchez Alvarado, A. 2003. Ingestion of bacterially expressed double-stranded RNA inhibits gene expression in planarians. Proc. Natl. Acad. Sci. (Suppl. 1) 100: 11861-11865.

Pasquinelli, A.E., Reinhart, B.J., Slack, F., Martindale, M.Q., Kuroda, M.I., Maller, B., Hayward, D.C., Ball, E.E., Degnan, B., Muller, P., et al. 2000. Conservation of the sequence and temporal expression of let-7 heterochronic regulatory RNA. Nature 408: 86-89.

Petersen, C.P., Bordeleau, M.E., Pelletier, J., and Sharp, P.A. 2006. Short RNAs repress translation after initiation in mammalian cells. Mol. Cell 21: 533-542.

Pillai, R.S., Bhattacharyya, S.N., Artus, C.G., Zoller, T., Cougot, N., Basyuk, E., Bertrand, E., and Filipowicz, W. 2005. Inhibition of translational initiation by let-7 microRNA in human cells. Science 309: 1573-1576.

Reddien, P.W., Bermange, A.L., Murfitt, K.J., Jennings, J.R., and Sánchez Alvarado, A. 2005a. Identification of genes needed for regeneration, stem cell function, and tissue homeostasis by systematic gene perturbation in planaria. Dev. Cell 8: 635-649.

Reddien, P.W., Oviedo, N.J., Jennings, J.R., Jenkin, J.C., and Sánchez Alvarado, A. 2005b. SMEDWI-2 is a PIWI-like protein that regulates planarian stem cells. Science 310: 1327-1330.

Reinhart, B.J., Slack, F.J., Basson, M., Pasquinelli, A.E., Bettinger, J.C., Rougvie, A.E., Horvitz, H.R., and Ruvkun, G. 2000. The 21nucleotide let-7 RNA regulates developmental timing in Caenorhabditis elegans. Nature 403: 901-906.

Rokas, A., Kruger, D., and Carroll, S.B. 2005. Animal evolution and the molecular signature of radiations compressed in time. Science 310: 1933-1938.

Sánchez Alvarado, A. 2006. Planarian regeneration: Its end is its beginning. Cell 124: 241-245.

Sánchez Alvarado, A., Newmark, P.A., Robb, S.M., and Juste, R. 2002. The Schmidtea mediterranea database as a molecular resource for studying platyhelminthes, stem cells and regeneration. Development 129: 5659-5665.

Schratt, G.M., Tuebing, F., Nigh, E.A., Kane, C.G., Sabatini, M.E., Kiebler, M., and Greenberg, M.E. 2006. A brain-specific microRNA regulates dendritic spine development. Nature 439: 283289.

Suh, M.R., Lee, Y., Kim, J.Y., Kim, S.K., Moon, S.H., Lee, J.Y., Cha, K.Y., Chung, H.M., Yoon, H.S., Moon, S.Y., et al. 2004. 
Human embryonic stem cells express a unique set of microRNAs. Dev. Biol. 270: 488-498.

Valencia-Sanchez, M.A., Liu, J., Hannon, G.J., and Parker, R. 2006. Control of translation and mRNA degradation by miRNAs and siRNAs. Genes \& Dev. 20: 515-524.

Xie, X., Lu, J., Kulbokas, E.J., Golub, T.R., Mootha, V., LindbladToh, K., Lander, E.S., and Kellis, M. 2005. Systematic discovery of regulatory motifs in human promoters and $3^{\prime}$ UTRs by comparison of several mammals. Nature 434: 338-345.
Yekta, S., Shih, I.H., and Bartel, D.P. 2004. MicroRNA-directed cleavage of HOXB8 mRNA. Science 304: 594-596.

Zayas, R.M., Hernandez, A., Habermann, B., Wang, Y., Stary, J.M., and Newmark, P.A. 2005. The planarian Schmidtea mediterranea as a model for epigenetic germ cell specification: Analysis of ESTs from the hermaphroditic strain. Proc. Natl. Acad. Sci. 102: 1849118496.

Zuker, M. 2003. Mfold web server for nucleic acid folding and hybridization prediction. Nucleic Acids Res. 31: 3406-3415. 

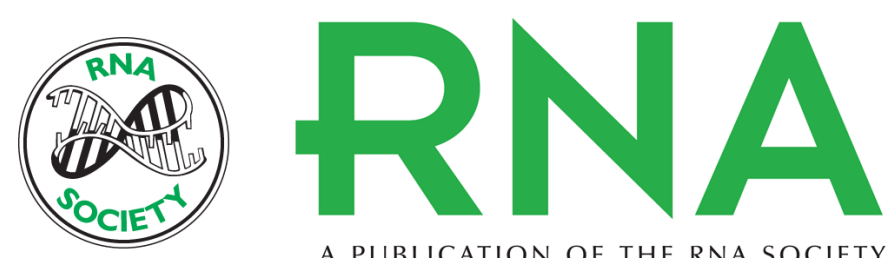

A PUBLICATION OF THE RNA SOCIETY

\section{MicroRNAs from the Planarian Schmidtea mediterranea: A model system for stem cell biology}

Dasaradhi Palakodeti, Magda Smielewska and Brenton R. Graveley

RNA 2006 12: 1640-1649

References This article cites 49 articles, 18 of which can be accessed free at:

http://rnajournal.cshlp.org/content/12/9/1640.full.html\#ref-list-1

License

Email Alerting Receive free email alerts when new articles cite this article - sign up in the box at the Service top right corner of the article or click here.

To subscribe to RNA go to:

http://rnajournal.cshlp.org/subscriptions 